\title{
Efectividad de la rehabilitación basada en la comunidad en preescolares con retraso del lenguaje expresivo
}

\author{
Effectiveness of community based rehabilitation in preschool children with language speech delay
}

Carlos José Fajardo 1,2 (iD https://orcid.org/0000-0002-6850-8308, Mercedes Martínez 3 (1) https://orcid.org/0000-0002-8408-5456

Sonia Flores Elvir ${ }^{5}$ (D) https://orcid.org/0000-0002-0187-3765.

\begin{abstract}
'Universidad Nacional Autónoma de Honduras (UNAH), Facultad de Ciencias Médicas, Departamento de Salud Pública, Maestría de Epidemiología.
${ }^{2}$ Centro de Cáncer Emma Romero de Callejas, Tegucigalpa, Honduras.

${ }^{3}$ Instituto Hondureño de Seguridad social, Departamento de Rehabilitación, Centro Especializado de Medicina Física y Rehabilitación.

${ }^{4}$ UNAH, Unidad Universitaria de Rehabilitación, Tegucigalpa, Honduras.

5Instituto Hondureño de Seguridad social, Departamento de Rehabilitación, Centro Especializado de Medicina Física y Rehabilitación, Tegucigalpa, Honduras.
\end{abstract}

RESUMEN. Antecedentes: En Honduras, $28 \%$ de los niños con discapacidad presenta alguna alteración en la función relacionada con la voz y el habla. La Rehabilitación Basada en la Comunidad (RBC) es una estrategia para mejorar el acceso a los servicios de rehabilitación. Objetivo: Comparar la efectividad de terapias de lenguaje con enfoque RBC versus terapia convencional en niños con Retraso en el Lenguaje Expresivo (RLE) en el Centro Especializado de Medicina Física y Rehabilitación, Instituto Hondureño de Seguridad Social, (IHSS), Honduras, junio 2019 enero 2020. Metodología: Estudio ensayo clínico aleatorizado. Se diagnosticaron niños de 2-5 años con RLE aleatorizados en grupo de intervención (RBC) y en grupo control; 28 niños participaron en terapias RBC y 20 en terapia convencional. La intervención consistió en capacitar a los padres para que realizaran terapias con sus hijos en casa. Se caracterizó la población y se realizaron modelos de regresión de Cox. Resultados: 48 pacientes completaron dos evaluaciones. La mediana de edad fue 3 años, $77.1 \%$ entre $2-3$ años, sexo masculino predominó con $79.2 \%$, procedencia urbana con $82.9 \%$, madres con nivel educativo superior $47.9 \%$. Los niños en RBC tuvieron $4.7 \%$ más probabilidad de mejorar Hazard Ratio (HR) crudo (1.047, $p=0.889$; IC 95\% 0.5434 - 2.0194); al ajustar la probabilidad de mejorar fue de $10.2 \%$ (HR ajustado 1.1016 , $p=0.783 ;$ IC95\% 0.55-2.1966). Ninguno de los modelos alcanzó significancia estadística. Discusión: No se identificó diferencia en la efectividad entre los grupos del estudio; utilizar terapias RBC puede disminuir costos para el derechohabiente y para el IHSS.

Palabras clave: Investigación participativa basada en la comunidad; Niños con discapacidad; Rehabilitación de los trastornos del habla y del lenguaje; Trastornos del desarrollo del lenguaje.
Recibido: 19-08-2021 Aceptado: 02-12-2021 Primera vez publicado en línea: 14-12-2021 Dirigir correspondencia a: Dr. Carlos José Fajardo

Correo electrónico: cjfajardo92@gmail.com

DECLARACIÓN DE RELACIONES Y ACTIVIDADES FINANCIERAS Y NO FINANCIERAS: Ninguna.

DECLARACIÓN DE CONFLICTOS DE INTERÉS: Ninguno.

\section{INTRODUCCIÓN}

La Discapacidad es un término que incluye déficit, limitaciones en la actividad y restricciones en la participación. ${ }^{1} \mathrm{Se}$ estima que la discapacidad infantil alcanza a 95 millones de niños, con una prevalencia mundial de $5.1 \% .^{2,3}$ Los trastornos del lenguaje expresivo tienen una prevalencia mundial estimada entre 5-15\% (mediana 6\%), con mayor incidencia en niños del sexo masculino en una razón de $3: 1 .^{4-6}$ En Colombia en 2014, se encontró una prevalencia de $1.86 \%$ en niños en edad preescolar; ${ }^{7}$ en cambio en Argentina en el año 2018, se encontró una prevalencia de $11.6 \%{ }^{8}$ En Honduras en el año 2017, se calculó una prevalencia de discapacidad infantil de $9.0 \%$; el $28 \%$ de estos presentaba alguna alteración en la función relacionada con la voz y el habla. ${ }^{9}$

Las personas con discapacidad experimentan niveles más deficientes de salud y se enfrentan a diversos retos para alcanzar sus derechos. ${ }^{10}$ La Rehabilitación Basada en la Comunidad (RBC) es una estrategia que mejora el acceso a los servicios de rehabilitación para personas con discapacidad, a través de la colaboración activa de dirigentes locales, familiares y otros actores clave haciendo uso de herramientas en su propia comunidad. ${ }^{2,11}$ Por otra parte, los Retrasos en el Lenguaje Expresivo (RLE) se definen como una deficiencia en la articulación de sonidos hablados, fluencia, comprensión o uso de los símbolos escritos. ${ }^{12,13}$ Para el manejo de estos retrasos es ampliamente reconocida la necesidad de intervenciones que incluyan la comunicación cotidiana de un compañero de hogar y que sea realizada en los ambientes naturales donde el niño se desarrolla. ${ }^{12}$ En la literatura se han descrito algunos factores de riesgo para

Forma de citar: Fajardo CJ, Martínez M, Flores Elvir S. Efectividad de la rehabilitación basada en la comunidad en preescolares con retraso del lenguaje expresivo. Rev Med Hond. 2021; 89 (2): 109-116. DOI: https://doi.org/10.5377/rmh.v89i2.12869

(C) 2021 Autor(es). Artículo de acceso abierto bajo la licencia https://creativecommons.org/ licenses/by/4.0/deed.es 
el RLE como ser: sexo masculino, bajo nivel educativo de la madre, número alto en el orden de nacimiento, historia familiar de RLE, entre otros. ${ }^{14}$ En cuanto al pronóstico, niños con RLE en años preescolares tienen un riesgo cuatro veces mayor de desarrollar discapacidades de aprendizaje en años escolares 0 incluso hasta en edad adulta. ${ }^{15,16}$ Los RLE son patologías que mediante intervenciones poco complejas y de corta duración, pueden mejorar clínicamente, siendo propicios para demostrar la efectividad de una terapia con enfoque de RBC.

En este estudio la intervención consistió en la capacitación de los padres en cuanto a la aplicación de diferentes estrategias para la mejora del lenguaje expresivo de sus hijos, con la intención que estos realizaran las terapias en casa. El grupo control continuó con sus terapias de forma ambulatoria en el Centro Especializado de Medicina Física (CEMFyR) y Rehabilitación del IHSS como se realiza de forma habitual. El objetivo de este estudio fue comparar la efectividad de ambas terapias en niños entre las edades de 2 a 5 años. La CEMFyR del IHSS pretende aumentar la cobertura de sus pacientes pediátricos con discapacidad mediante el enfoque RBC. La intención pragmática de este estudio tiene como fin facilitar la implementación de sus resultados en la práctica diaria. Esta estrategia de RBC beneficiaría a los derechohabientes, que disminuirían gastos de traslado, un menor ausentismo laboral, escolar, y una menor saturación de los servicios hospitalarios.

\section{PARTICIPANTES Y MÉTODOS}

Se realizó un estudio tipo ensayo clínico aleatorizado con intención pragmática. ${ }^{17}$ El universo fue niños de 2 a 5 años evaluados en el CEMFyR a partir del mes de junio 2019 hasta enero 2020. Los criterios de inclusión: niños entre 2 y 5 años con RLE diagnosticado por médico especialista en Audiología y Foniatría en el momento de la captación, no haber recibido ninguna terapia previa al período de intervención, nacimiento a término sin ninguna complicación en el parto, no tener alguna discapacidad intelectual y que la lengua materna sea el español. Se excluyeron los niños en los que la terapia hospitalaria era obligatoria por cualquier indicación.

Al momento del diseño del protocolo, se tomó como parámetro la muestra calculada en el ensayo clínico de Roberts y colaboradores. ${ }^{18}$ En dicho estudio se determinó que se podría detectar una diferencia de 0.43 desviaciones estandar con 90 niños (45 en cada brazo) con un poder de $80 \%$ y nivel de significancia de $5 \%$. Partiendo de esto se estimó un tamaño muestral de 97 niños, 45 en el grupo de intervención (RBC) y 52 en el grupo control (clínica ambulatoria). La captación de niños inició en julio 2019 y estaba previsto continuar hasta alcanzar la muestra calculada. Con la declaratoria de emergencia nacional por la pandemia COVID-19, en marzo 2020 se suspendió la captación de nuevos niños y las terapias de aquellos que no habían concluido. Con los niños que ya habían completado dos evaluaciones, se recalculó el poder estadístico con un nivel de confianza de $95 \%$, tamaño de efecto de g:0.42 y una desviación estándar de $0.43,{ }^{18}$ y se logró un poder estadístico de $91.2 \%$, el número de participantes quedo conformado 48 niños en total, 28 niños en el grupo RBC y 20 niños en el grupo control.

La captación y la aleatorización se realizaron en un mismo momento. Los niños incluidos fueron clasificados en una de las siguientes categorías ordinales: Palabras sueltas (normal para un año), palabras yuxtapuestas (normal para dos años), frases cortas de 3 palabras (normal para 3 años), oraciones más largas con problemas de estructuración (normal para 4 años), oraciones bien estructuradas y problemas para pronunciar la $/ \mathrm{rr} / \mathrm{rl}$ (normal para 5 años), siendo RLE cuando el niño fue clasificado en una categoría inferior a la que correspondía según su edad. Se comparó la proporción de subcategorías de diagnóstico de RLE en ambos grupos para garantizar que los grupos fuesen comparables en relación con los diagnósticos de sus integrantes.

Inmediatamente al diagnóstico de cada niño, la médica foniatra informó la captación vía telefónica al investigador encargado de la aleatorización. Este último asignó un número correlativo a cada niño generado al azar del 1 al 97 (https://www. random.org/) y se enlistó según el orden de captación, los pares se asignaban al grupo control y los impares se incluía en el grupo RBC. Al salir de la primera evaluación cada niño ya tenía asignado el tipo de terapia que recibiría. Debido al tipo de patología evaluada, las características intrínsecas de la terapia y el personal de salud disponible, el cegamiento del médico evaluador, los pacientes y de sus padres no fue posible.

La recolección de los datos se realizó mediante un instrumento de observación directa basado en el Expressive OneWord Picture Vocabulary Test, Fourth Edition y adaptado al español por la Lic. Linna Reyes y su equipo del Servicio de Terapia de Habla y Lenguaje de la Unidad Universitaria de Medicina de Rehabilitación, UNAH.

El instrumento constaba de los siguientes apartados: características sociodemográficas (edad y sexo del niño, lugar de residencia, nivel educativo de la madre, años de escolaridad de los padres, ubicación en el orden de nacimiento del niño en relación a sus hermanos) prerrequisitos lingüísticos (atención, imitación, permanencia de objetos, medios fines), lenguaje expresivo (vocabulario, nombra objetos por categoría, nombra relaciones espaciales, responde preguntas, definición de palabras comunes, etc.), articulación de palabras, mecanismo oral por medio de evaluación física, evaluación del ritmo (repeticiones, fijaciones, pausas, prolongaciones, movimientos secundarios) y evaluación de la voz (clavicular, torácica, torácica diafragmática). Este instrumento es el que se utiliza de forma rutinaria en el servicio de foniatría del CEMFyR, por lo tanto, no requirió validación; se aplicó para diagnosticar a los niños con RLE previo y para medir el grado de mejoría posterior a la intervención.

La segunda evaluación se realizó ocho semanas después del inicio de las terapias y según el resultado se reclasificó en las categorías de diagnósticos. Se consideró mejoría si el niño es reclasificado en la categoría de diagnóstico normal para su edad - a una categoría más cercana a la esperada para su edad.

Tanto las terapias RBC como las terapias clínicas ambulatorias se basaron en ejercicios orofaciales, lenguaje comprensivo y estructura de la oración. Las principales diferencias entre 
las terapias fueron 1) las terapias RBC fueron realizadas por los padres de los niños en su domicilio (siendo previamente entrenados en una única capacitación de 3 horas impartida por un terapista del lenguaje) y las terapias del grupo control fueron impartidas por los terapistas del lenguaje en la clínica ambulatoria 2) los de RBC tuvieron un seguimiento sin aplicación de instrumento en el hospital a las 4 semanas y la segunda evaluación a las 8 semanas; en cambio los niños en el grupo control, recibieron sus terapias en la clínica ambulatoria una vez por semana por las 8 semanas. Específicamente, las estrategias instruidas a los padres fueron: nominación de figuras de animales y sus onomatopeyas correspondientes; identificar y nominar partes del cuerpo, prendas de vestir, familiares u objetos de su interés cuando los niños solo expresaban monosílabos; descripción de las funciones y las acciones de objetos, figuras y situaciones de la vida diaria en niños que solo pronunciaban palabras yuxtapuestas. Cuando el niño omitía sílabas se trabajó con figuras en ejercicios de síntesis silábica, hasta que el niño articulaba la palabra completa. Además, se enseñaron a los padres los ejercicios orofaciales (ejercicios de lengua, labios y paladar) para realizar en casa. Cuando los niños solo omitían letras se realizaron terapias para lograr punto y modo de articulación de cada letra. Cuando el niño decía oraciones pero no las estructuraban adecuadamente, se trabajó en descripción de lámina.

Las descripciones de lámina consistían en pedirle al niño que describiera situaciones de la vida diaria, con cuestiones cotidianas a ellos, esto para lograr el uso adecuado de conexivos en las oraciones. ${ }^{19}$

Posterior a las capacitaciones brindadas a los padres de ambos grupos, uno por cada niño completó un instrumento adicional donde se midió el grado de comprensión, compromiso, actitud y aptitud hacia el proceso de rehabilitación. Cada categoría se evaluó en una escala de Likert del 1-5, conformando un total de 18 preguntas: 4 de comprensión, 3 de compromiso, 5 de aptitud y 6 de actitud. Se calculó el promedio de todas las respuestas y se clasificó en malo si el puntaje era de 1-2, regular si el puntaje era 3 y bueno entre 4-5. El objetivo de esta medición era determinar si el desempeño de los padres influye en beneficio de alguna de las terapias.

Se ingresaron los datos a una base en Excel versión 16.30. La calidad de la digitación de los datos, la verificación de consistencia y proceso de limpieza de los mismos estuvo a cargo del investigador principal. El análisis se realizó usando el paquete estadístico STATA 14.2. Con la muestra total de niños, se aplicaron las pruebas de normalidad determinando el comportamiento no normal de la muestra. Para las variables cuantitativas se calculó la mediana y prueba de Wilcoxon para muestras independientes; para las variables cualitativas se utilizaron frecuencias, porcentajes y se calcularon las diferencias de proporciones entre cada uno de los grupos de estudio y se calculó el Chi cuadrado para medir la asociación entre las edades y las subcategorías de diagnóstico.

Se creó una variable dependiente dicotómica que consideró mejoría o sin mejoría, según lo descrito anteriormente. También, se creó la variable de tiempo que midió el número de semanas que los niños recibieron las terapias. Se realizó un modelo de regresión de Cox univariado que midió la probabilidad de mejorar en el grupo RBC en comparación al control mediante el Hazard Ratio (HR) crudo, junto con el intervalo de confianza (IC) al $95 \%$ y el valor de $p$. Además, se realizó un modelo multivariado ajustando el HR por sexo, edad, nivel educativo de la madre, procedencia y orden de nacimiento de los niños, junto con el IC al $95 \%$ y valor de $p$. Para el análisis de la comprensión, compromiso, aptitud y actitud de los padres hacia las terapias, se calcularon las proporciones y las diferencias de proporciones para cada aspecto evaluado. Se hizo un análisis de supervivencia de Kaplan-Meyer para medir el evento: diagnóstico de RLE donde se tomaron en cuenta 77 niños que cumplieron los criterios de inclusión, independientemente si culminaron o no las terapias; esto con el objetivo de medir la probabilidad de ser diagnosticado por cada edad entre los 2 y los 5 años. Para esto se creó una variable cuantitativa discreta que midió el tiempo en años (cumplidos) desde la fecha de nacimiento a la fecha de diagnóstico de RLE.

El protocolo fue aprobado por el equipo docente de la Maestría de Epidemiología de la Facultad de Ciencias Médicas de la UNAH. La aprobación ética se obtuvo del Comité de Bioética del departamento de Gerencia de Docencia e Investigación del IHSS en Tegucigalpa, Honduras; mediante el Acta 085-CB-HE. Una vez obtenida dicha aprobación, se inició el reclutamiento de niños con sospecha de RLE que asistían de forma espontánea o referidos al área de Foniatría del CEMFyR.

Se solicitó por escrito un consentimiento informado a los padres. Se enfatizó que la participación en el estudio era voluntaria y que podrían retirarse del mismo en cualquier momento sin ninguna consecuencia para la atención del niño. Además, se enfatizó que los padres no iban a recibir ningún beneficio económico por su participación y que el beneficio de participar sería el diagnóstico oportuno y el tratamiento de su hijo. La información fue manejada de forma confidencial. Se tomaron como principios los presentados por The Global Health Network en el curso "Introducción a las responsabilidades de los investigadores en cuanto a las buenas prácticas clínicas" (2018).

\section{RESULTADOS}

Un total de 77 niños cumplieron los criterios de ingreso. El 53.2\% (41/77) fueron aleatorizados al grupo RBC y $46.8 \%$ (36/77) fueron aleatorizados al grupo control. 48/77 niños cumplieron con las terapias y las dos evaluaciones, $58.3 \%(28 / 48)$ del grupo RBC y $41.7 \%$ (20/48) del grupo control. Hubo 5 pérdidas, 3 en RBC y 2 en control (Figura 1).

En cuanto a las características epidemiológicas de los niños que completaron sus dos evaluaciones se encontró que $77.1 \%$ (37/48) era menor de 3 años, con mediana de edad de 3 años, $79.2 \%$ (38/48) fue del sexo masculino, $82.9 \%$ (39/48) procedían del área urbana, en el $47.9 \%$ (23/48) y $39.6 \%$ (19/48) el nivel educativo de la madre fue superior y medio, respectivamente, con una media de escolaridad de los padres de 13 años para el grupo de RBC y 11.5 años para el grupo 


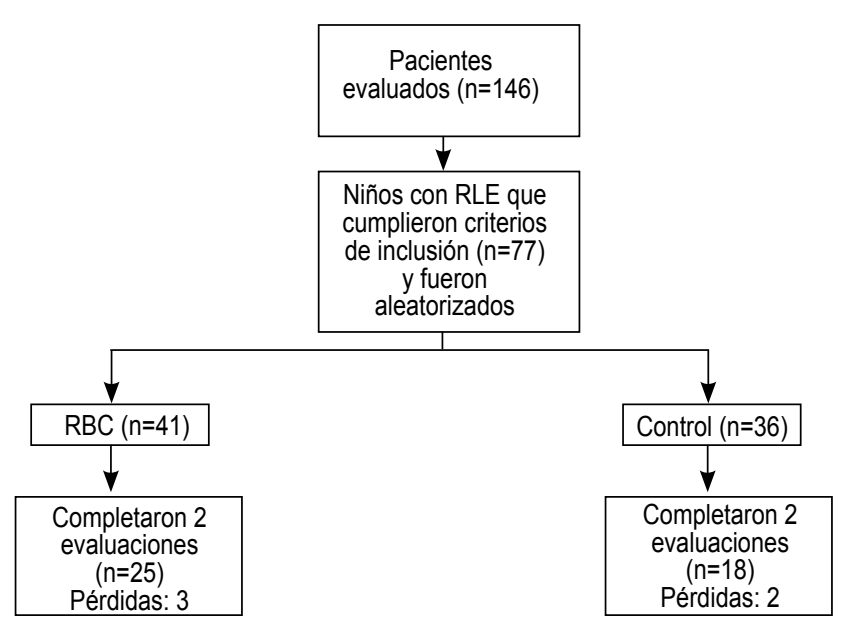

Figura 1. Diagrama de flujo de los niños participantes en el estudio. control. El 46.8\% (22/48) eran primogénitos. No hubo diferencias estadísticamente significativas en las proporciones de estas variables en los dos grupos del estudio (Cuadro 1).

En los diagnósticos encontrados a la captación, 52.1\% (25/48) correspondía a palabras sueltas, $14.6 \%$ (7/48) a palabras yuxtapuestas y $12.5 \%$ (6/48) a oraciones bien estructuradas, monosílabos y onomatopeyas. Mediante la prueba de chi cuadrado se encontró una asociación entre las variables edad y categoría de diagnóstico al momento de la captación $(p=0.000)$. Todas las subcategorías de diagnósticos de RLE tuvieron la misma proporción en ambos grupos de intervención (Cuadro 2).

El 75\% (36/48) de los niños mejoraron. De los niños que recibieron terapia RBC, el $80.0 \%$ (20/25) presentaron una mejoría objetiva, con 3 pérdidas que completaron sus terapias, pero no asistieron a su evaluación. Del grupo control mejoraron el $88.9 \%$ (16/18), con 2 pérdidas.

Cuadro 1. Características sociodemográficas de los niños de 2-5 años con RLE distribuidos según el grupo de intervención al que fueron asignados. Servicio CEMFyR del IHSS, Tegucigalpa Honduras, junio 2019 - enero 2020.

\begin{tabular}{|c|c|c|c|c|c|c|}
\hline \multirow[t]{3}{*}{ Variable } & \multicolumn{4}{|c|}{ Grupo } & \multirow{3}{*}{$\left(\right.$ IC $\left.95 \%{ }^{++}\right)$} & \multirow{3}{*}{ valor de $p^{+++}$} \\
\hline & \multirow{2}{*}{$\begin{array}{c}\text { Total } \\
(\mathrm{n}=48) \\
\mathrm{n}(\%)\end{array}$} & \multirow{2}{*}{$\begin{array}{c}\text { RBC } \\
(n=28) \\
n(\%)\end{array}$} & $\begin{array}{l}\text { Control } \\
(n=20)\end{array}$ & \multirow[t]{2}{*}{$\begin{array}{l}\text { Diferencia de } \\
\text { proporciones }^{+}\end{array}$} & & \\
\hline & & & $\mathrm{n}(\%)$ & & & \\
\hline \multicolumn{7}{|l|}{ Edad (años) } \\
\hline 2 & $18(37.5)$ & $11(39.3)$ & $7(35.0)$ & 4.00 & $(-38.9-54.9)$ & 0.742 \\
\hline 3 & $19(39.6)$ & $11(39.3)$ & $8(40.0)$ & -1.00 & $(-47.7-41.7)$ & 0.895 \\
\hline 4 & $3(6.3)$ & $1(3.6)$ & $2(10.0)$ & -7.00 & $(-34.8-20.8)$ & 0.676 \\
\hline 5 & $8(16.6)$ & $5(17.9)$ & $3(15.0)$ & 12.60 & $(-29.3-54.4)$ & 0.608 \\
\hline Mediana de edad & & 3 & 3 & & & $0.789^{*}$ \\
\hline \multicolumn{7}{|l|}{ Sexo } \\
\hline Masculino & $38(79.2)$ & $22(78.6)$ & $16(80.0)$ & -0.30 & $(-27.1-26.5)$ & 0.982 \\
\hline Femenino & $10(20.8)$ & $6(21.4)$ & $4(20.0)$ & 0.40 & $(-51.2-52.0)$ & 0.987 \\
\hline \multicolumn{7}{|l|}{ Procedencia } \\
\hline Urbano & $39(82.9)$ & $22(78.6)$ & $18(90.0)$ & -10.90 & $(-33.4-11.6)$ & 0.366 \\
\hline Rural & $8(17.2)$ & $6(21.4)$ & $2(10.0)$ & -10.90 & $(-42.7-64.5)$ & 0.731 \\
\hline \multicolumn{7}{|l|}{ Nivel educativo de la madre } \\
\hline Nivel bajo & $6(12.5)$ & $3(10.7)$ & $3(10.7)$ & 0.20 & $(-54.2-55.2)$ & 0.994 \\
\hline Nivel Medio & $19(39.6)$ & $11(39.3)$ & $8(40.0)$ & -2.90 & $(-47.6-41.8)$ & 0.898 \\
\hline Nivel Superior & $23(47.9)$ & $14(50.0)$ & $9(45.0)$ & 2.70 & $(-39.1-44.5)$ & 0.899 \\
\hline Años de escolaridad de padres^ ${ }^{\wedge}$ & & 13 & 11.5 & & & $0.204^{*}$ \\
\hline \multicolumn{7}{|l|}{ Orden de Nacimiento } \\
\hline Primero & $22(46.8)$ & $11(39.3)$ & $12(60.0)$ & -18.90 & $(-59.6-22.4)$ & 0.3828 \\
\hline Segundo & $18(38.3)$ & $12(42.9)$ & $6(0.3)$ & 11.30 & $(-35.2-57.8)$ & 0.6432 \\
\hline Tercero & $7(14.9)$ & $5(17.9)$ & $2(10.0)$ & 7.30 & $(-46.8-61.4)$ & 0.8105 \\
\hline
\end{tabular}

+Puntos porcentuales

$++\mid$ C95\% de la diferencia de proporciones

+++Para diferencias de proporciones (valor $\mathrm{z}$ )

*Wilcoxon para dos muestras independientes (U Mann Whitney)

^Mediana 
Cuadro 2. Diagnósticos de los niños de 2-5 años con RLE distribuidos según grupo de intervención al que fueron asignados. Servicio de CEMFyR del IHSS, Tegucigalpa Honduras, julio 2019 - enero 2020.

\begin{tabular}{|c|c|c|c|c|c|c|}
\hline \multirow[b]{2}{*}{ Categorías de diagnóstico a la captación } & \multirow[b]{2}{*}{$\begin{array}{c}\text { Total } \\
(\mathrm{n}=48) \\
\mathrm{n}(\%)\end{array}$} & \multicolumn{2}{|c|}{ Grupo } & \multirow[b]{2}{*}{$\begin{array}{c}\text { Diferencia de } \\
\text { proporciones }^{+}\end{array}$} & \multirow[b]{2}{*}{$\left(\mathrm{IC} 95 \%{ }^{++}\right)$} & \multirow[b]{2}{*}{ Valor de $p^{+++}$} \\
\hline & & $\begin{array}{c}\text { RBC } \\
(n=28) \\
n(\%)\end{array}$ & $\begin{array}{c}\text { Control } \\
(n=20) \\
n(\%)\end{array}$ & & & \\
\hline Monosílabos y onomatopeyas & $6(12.5)$ & $4(14.3)$ & $2(10.0)$ & 4.3 & $(-49.6-58.2)$ & 0.882 \\
\hline Palabras sueltas & $25(52.1)$ & $14(50.0)$ & $11(55.0)$ & -5 & $(-44.4-34.4)$ & 0.803 \\
\hline Palabras yuxtapuestas & $7(14.6)$ & $3(10.7)$ & $4(20.0)$ & -9.3 & $(-61.8-43.3)$ & 0.739 \\
\hline Frases cortas & $3(6.3)$ & $2(7.14)$ & $1(5.0)$ & 0.02 & $(-61.8-43.2)$ & 0.944 \\
\hline Problemas de estructuración de la oración y las letras/ SIL/. & $2(4.1)$ & $1(3.6)$ & $1(5.0)$ & -1.43 & $(-41.1-38.3)$ & 0.945 \\
\hline Oraciones bien estructuradas y problemas solo con la /r/rrl. & $5(10.4)$ & $4(14.3)$ & $1(5.0)$ & 9.3 & $(-45.5-64.1)$ & 0.801 \\
\hline
\end{tabular}

+ Puntos porcentuales

++ Para diferencias de proporciones (valor z)

+++ IC95\% de la diferencia de proporciones

Se encontró que los niños en el grupo RBC tuvieron $4.7 \%$ más probabilidad de mejorar en comparación con los niños en el grupo control, sin embargo, no se encontró una diferencia estadísticamente significativa (HR crudo 1.047, $p=0.889$; IC95\% $0.5434-2.0194)$. Al realizar un modelo multivariado, se encontró que los niños en el grupo RBC tuvieron $10.2 \%$ más probabilidad de mejorar (HR ajustado, 1.1016, $p=0.783$; IC95\% 0.55 - 2.1966) al ajustar el riesgo por edad de los niños, sexo, lugar de procedencia, nivel educativo de la madre y orden de nacimiento, sin encontrar significancia estadística (Cuadro 3).

El nivel de comprensión de la terapia, compromiso aptitud y actitud por parte de los padres fue bueno en $88.3 \%$ (68/77), $87.0 \%(67 / 77) 93.5 \%$ (72/77) y $85.7 \%$ (66/77), respectivamente en ambos grupos. No se encontraron diferencias estadísticamente significativas en cuanto a los niveles de comprensión, compromiso, actitud y aptitud de los padres entre los grupos a los que los niños con RLE fueron asignados (Cuadro 4)

De los 77 niños estudiados $48 \%$ (37/77) fueron diagnosticados a los 2 años, $75 \%$ (58/77) a los 3 años o antes, $10 \%$ (8/77) a los 4 años y 15\% (11/77) a los 5 años.

Cuadro 3. Cuadro de la probabilidad de mejoría (HR) en las terapias RBC según los modelos univariado y multivariado de los niños de 2-5 años. Servicio de CEMFyR del IHSS, Tegucigalpa Honduras, julio 2019-enero 2020.

\begin{tabular}{lccc}
\hline \multicolumn{1}{c}{ Variables } & HR $^{*}$ & valor de $\boldsymbol{p}$ & (IC95\%) \\
\hline $\begin{array}{l}\text { Modelo Univariado } \\
\text { RBC }\end{array}$ & 1.047 & 0.889 & $(0.543-2.019)$ \\
Modelo Multivariado & & & \\
RBC & 1.1016 & 0.783 & $(0.552-2.196)$ \\
Edad & 0.9128 & 0.588 & $(0.656-1.269)$ \\
Sexo & 1.2098 & 0.675 & $(0.497-2.943)$ \\
Lugar de procedencia & 1.0552 & 0.908 & $(0.424-2.621)$ \\
Nivel educativo de la madre & 1.012 & 0.919 & $(0.796-1.288)$ \\
Orden de nacimiento & 0.8447 & 0.527 & $(0.500-1.425)$ \\
\hline
\end{tabular}

*HR (Hazard ratio, cociente de riesgos instantáneos)

\section{DISCUSIÓN}

Las probabilidades de mejorar en su RLE en el grupo RBC en comparación con el grupo control fue de $10.2 \%$ al ajustar por edad, sexo, procedencia, nivel educativo de la madre y orden de nacimiento. Esto coincide con los demostrado en un ensayo clínico aleatorizado de EE. UU. en el 2015 por Roberts y colaboradores, donde la intervención consistió en capacitar a los padres en los métodos para que ellos mismos impartieran las terapias a sus hijos frente a las terapias por personal de salud midiendo la diferencia de medias de las puntuaciones en la prueba Preschool Language Scale, Fourth Edition, cuyo intervalo de confianza (-4.5 a 5.3) y valor de $p(0.88)$, no alcanzando significancia estadística. ${ }^{18}$

Estos resultados también coinciden con un metaanálisis realizado por Roberts M y colaboradores en 2011, en donde no se encontraron diferencias significativas en cuanto al tamaño de efecto $(g=0.25$, IC $95 \%[-0.43,0.93]$, valor de $p=0.47)$ en las habilidades expresivas de los niños que recibieron terapias de sus padres en comparación a los niños que las recibieron de terapistas de lenguaje; aunque sí encontraron una mejoría significativa en habilidades receptivas $(g=0.41$, IC $95 \%$ [0.08, $0.76]$, valor de $p=0.02$ ) de los niños que se sometieron a las terapias por personal de salud. ${ }^{20}$ Las habilidades receptivas no fueron evaluadas en este estudio. Otro metaanálisis realizado por Law y colaboradores en 2004 que incluyó 25 estudios de intervención demostró que no había diferencias significativas entre las terapias cuando las terapias tenían una duración no menor a 8 semanas como fue el caso de este estudio. ${ }^{21}$

Los padres mostraron un grado de compromiso, comprensión, actitud y aptitud de $88,87,94,86 \%$ respectivamente en ambos grupos, no mostrando una diferencia estadísticamente significativa al hacer una diferencia de proporciones entre grupos. Esto es comparable con los resultados del metaanálisis por Roberts y colaboradores en 2011, donde los padres que reciben capacitación presentaron mejores resultados (tamaños de efecto con valores positivos y significativos) en cuanto al interés 
Cuadro 4. Nivel de comprensión, compromiso, aptitud y actitud de los padres de niños con RLE, distribuidos según el tipo de terapia recibida por sus hijos. Servicio CEMFyR, junio 2019 - enero 2020.

\begin{tabular}{|c|c|c|c|c|c|c|}
\hline \multirow[b]{2}{*}{ Evaluación de padres } & \multicolumn{4}{|c|}{ Grupo } & \multirow[b]{2}{*}{ valor de $p^{++}$} & \multirow[b]{2}{*}{$\left(\right.$ IC95\% $\left.{ }^{+++}\right)$} \\
\hline & $\begin{array}{l}\text { Total } \\
\mathrm{n}=77 \\
\mathrm{n}(\%)\end{array}$ & $\begin{array}{l}R B C \\
n=39 \\
n(\%)\end{array}$ & $\begin{array}{c}\text { Control } \\
n=38 \\
n(\%)\end{array}$ & $\begin{array}{l}\text { Diferencia de } \\
\text { proporciones }^{+}\end{array}$ & & \\
\hline \multicolumn{7}{|l|}{ Comprensión } \\
\hline Buena & $68(88.3)$ & $34(50.0)$ & $34(50.0)$ & 0.00 & 1.0000 & $(-23.7-23.7)$ \\
\hline Regular & $7(9.1)$ & $4(57.1)$ & $3(42.9)$ & 14.2 & 0.7100 & $(-59.8-88.2)$ \\
\hline Mala & $2(2.6)$ & $1(50.0)$ & $1(50.0)$ & 0.00 & 1.0000 & $(-97.9-97.9)$ \\
\hline \multicolumn{7}{|l|}{ Compromiso } \\
\hline Bueno & $67(87.0)$ & $34(50.7)$ & $33(49.3)$ & 1.40 & 0.9088 & $(-22.5-25.3)$ \\
\hline Regular & $8(10.4)$ & $4(50.0)$ & $4(50.0)$ & 0.00 & 1.0000 & $(-69.2-69.2)$ \\
\hline Malo & $2(2.6)$ & $1(50.0)$ & $1(50.0)$ & 0.00 & 1.0000 & $(-97.9-97.9)$ \\
\hline \multicolumn{7}{|l|}{ Aptitud } \\
\hline Buena & $72(93.5)$ & $35(48.6)$ & $37(51.4)$ & -2.80 & 0.8123 & $(-25.8-20.2)$ \\
\hline Regular & $3(3.9)$ & $3(100)$ & $0(0)$ & & & \\
\hline Mala & $2(2.6)$ & $1(50.0)$ & $1(50.0)$ & 0.00 & 1.0000 & $(-97.9-97.9)$ \\
\hline \multicolumn{7}{|l|}{ Actitud } \\
\hline Buena & $66(85.7)$ & $33(50.0)$ & $33(50.0)$ & 0.00 & 1.0000 & $(-24.1-24.1)$ \\
\hline Regular & $9(11.7)$ & $5(55.6)$ & $4(44.4)$ & & & \\
\hline Mala & $2(2.6)$ & $1(50.0)$ & $1(50.0)$ & 0.00 & 1.0000 & $(-97.9-97.9)$ \\
\hline
\end{tabular}

mostrado hacia las terapias de sus hijos y un mayor repertorio de estrategias utilizadas para realizar la terapia, comparado a aquellos que no son capacitados, mostrando una diferencia significativa. ${ }^{20}$ Consideramos que los aspectos evaluados no influyen a favor de la eficacia de la terapia en ninguno de los grupos de intervención. En el ensayo clínico aleatorizado de Roberts y colaboradores, el nivel de estrés mostrado por los padres no mostró diferencias estadísticamente significativas, independientemente del grupo al que fueron asignados. ${ }^{18}$

Los niños de 2 y 3 años representaron el $75 \%$ de los diagnosticados con RLE, con una mediana de edad de 3 años. A través de un análisis de Kaplan-Meier se mostró las probabilidades de ser diagnosticado con un RLE, en donde los niños de 2 años tuvieron las probabilidades más altas de diagnóstico, seguido de los de 3, 5 y 4 años, respectivamente. En el metaanálisis de Roberts 2011 se encontró que más del $50 \%$ de 18 estudios tuvieron una mediana de edad entre 24 y 35 meses. ${ }^{20}$ Estos resultados demuestran que las edades entre 2 y 3 años son las más propicias para el diagnóstico de RLE, ya que en edades más tempranas los padres son capaces de detectar un retraso en la expresividad y buscar la atención médica, además los niños que son intervenidos a edades más tempranas tienen menos riesgo de secuelas a largo plazo..$^{15}$ En un estudio de cohorte retrospectivo realizado por Dale P. y colaboradores en el año 2014 se encontró que el $75.5 \%$ de los niños de 2 años con RLE ya no mostraba deficiencias a los 4 años incluso sin tratamiento, estableciendo que este diagnóstico es más difícil de realizar a medida se acerca a los 5 años debido a que la expresividad se nivela con sus contemporáneos. ${ }^{15}$ Aunque exista una mejoría en las habilidades expresivas cuando los niños no son tratados, existe a un riesgo 4 a 5 veces mayor de rendimientos deficientes a nivel escolar e incluso en la vida adulta cuando no son tratados. ${ }^{15,21}$

En cuanto a las demás características demográficas se encontró un marcado predominio en el sexo masculino, lo cual coincide con los resultados encontrados en una revisión sistemática de Berkman y colaboradores en 2015, en donde el hecho de ser hombre se encontró como un factor de riesgo en 11 de 14 estudios en idioma inglés que evaluaron RLE. ${ }^{22}$ En este estudio predominó el nivel superior educativo de los padres seguido del nivel medio, esto representa la población de derechohabientes del IHSS. Esto difiere de lo encontrado en la revisión sistemática de Berkman y colaboradores en el 2015, donde se encontró que un factor de riesgo para el desarrollo de RLE fue el nivel educativo bajo de los padres. ${ }^{22}$ Otro factor de riesgo que ha sido estudiado es la ubicación en el orden de nacimiento del niño, en este estudio los niños primogénitos fueron los más frecuentes; esto difiere de lo encontrado por Buschmann y colaboradores donde los niños que nacieron de segundo fueron los más frecuentes, aunque sin alcanzar significancia estadística. ${ }^{23}$ Esta variable no ha sido comprobada como factor de riesgo para RLE en la literatura. ${ }^{5,14,15,22}$ Hay un clara asociación estadística entre el tipo de diagnóstico de RLE y la edad del niño, ya que se mostró que estos niños están en la mayoría de los casos 1 ó 2 niveles por debajo del desarrollo normal para la edad coincidiendo con lo descrito en la literatura. ${ }^{6}$

Entre las limitantes encontradas en este estudio, el no cegamiento pudo haber introducido un sesgo de información, esto debido al tipo de diseño planteado, al escaso personal disponible para hacer el diagnóstico, el tipo de terapias y la médica que 
hizo las evaluaciones. Una estrategia para mitigar el posible sesgo fue la aleatorización de los participantes por un médico que no formaba parte del equipo clínico. Por otra parte, la declaración de emergencia Nacional por la epidemia COVID-19 imposibilitó un tamaño de muestra mayor. ${ }^{24}$

Este estudio provee evidencia que la RBC puede mejorar el RLE en niños de 2 a 5 años tanto como las terapias ambulatorias. Estos resultados podrían resultar ser prometedores tomando en cuenta las importantes ventajas clínicas y logísticas que ofrece el enfoque RBC, como ser la corta duración de las terapias y la reducción de costos del tratamiento, respectivamente, tanto para los pacientes y familiares como para el IHSS. Es necesario continuar estudiando la eficacia de las terapias $\mathrm{RBC}$ en poblaciones de menor nivel educativo que permita una generalización de los resultados. Además, se debe estudiar la eficacia de las terapias RBC para otras patologías que actualmente se manejan de forma ambulatoria.

\section{CONTRIBUCIONES}

Los autores declaramos que hemos participado suficientemente en la preparación del artículo, la revisión de la información sobre el diseño metodológico, los aspectos éticos de la investigación, la recolección y su análisis, la estructura del manuscrito y aprobación de la versión final para asumir la responsabilidad de su contenido. CF fue el investigador principal y participó activamente en la concepción de la idea de investi- gación, recolección, procesamiento y análisis estadístico de los datos. MM participó activamente el diagnóstico y seguimiento de los pacientes y el análisis clínico de los datos con enfoque a los conceptos de Foniatría. SF participó activamente en el análisis clínico de los datos con enfoque en los conceptos de Rehabilitación y RBC.

\section{AGRADECIMIENTOS}

A la Lic. Annie Núñez, Psicóloga, capacitada en terapia de Lenguaje por brindar la terapia a todos los niños y padres que participaron en el estudio. A la Lic. Diana Amador, psicóloga, por su colaboración en la agenda de niños para las terapias ambulatorias y para las charlas de capacitación de padres. A los docentes de la Maestría de Epidemiología de la UNAH, Dr. Mario Mejía, Dra. María Félix Rivera, Dra. Dilcia Sauceda, Dr. Jeremías Soto, Lic. Marlon Meléndez, Ing. Rafael Maradiaga $\mathrm{PhD}$ y a quien corresponde al Dr. Bomar Méndez por su asesoría metodológica.

\section{DETALLES DE AUTOR(ES)}

Carlos José Fajardo, Médico, Máster en Epidemiología; cjfajard092@gmail.com

Mercedes Martínez, Médica Especialista en Foniatría; melimague@yahoo.com.mx

Sonia Flores Elvir, Médica Especialista en Rehabilitación; soflorel62@gmail.com

\section{REFERENCIAS}

1. Organización Panamericana de la Salud. Organización Mundial de la Salud. Clasificación Internacional del funcionamiento, de la discapacidad y de la salud (cif). Versión abreviada. [Internet]. España: Red Española para la Clasificación y Evaluación de la Discapacidad(RECEDIS), I Instituto de Migraciones y Servicios Sociales (IMSERSO), 2001 [citado marzo 2020]. Disponible en: https://apps.who.int/iris/bitstream/handle/10665/43360/9241545445_spa.pdf;sequence=1

2. Organización Mundial de la Salud. Informe mundial sobre la discapacidad. [Internet]. Washington D.C: OMS; 2011. [citado marzo 2020];388. Disponible en: https://www.who.int/disabilities/world_report/2011/summary_es.pdf

3. Puga C, Pagotto V, Giunta D, Vicens J, Leist M, Vaucheret E, et al. Prevalencia e incidencia de discapacidad a partir del Certificado Único de Discapacidad en un hospital universitario del Área Metropolitana de Buenos Aires. Arch Argent Pediatr [Internet] 2019 [citado noviembre 2020];117(3):183-187. Disponible en: https://www.sap.org.ar/docs/publicaciones/archivosarg/2019/v117n3a13.pdf

4. Barragan-Perez E, Álvarez-Amado D, García-Beristain JC, Garfias-Rau C, Peinador Oliva M, Ladron de Guevara M, et al. Midiendo el espectro: de los trastornos del lenguaje a los trastornos del espectro autista: Consenso Latinoamericano Delphi Modificado. Rev Med Clin Condes. [Internet] 2021 [citado marzo 2021]; 32(1) 112-127. Disponible en: https://www.sciencedirect.com/science/article/pii/S071686402100002X

5. Wallace IF, Berkman ND, Watson LR, Coyne-Beasley T, Wood CT, Cullen $\mathrm{K}$, et al. Screening for Speech and language delay in children 5 years old and younger: a systematic review. Pediatrics. [Internet]. 2015 [citado abril 2020];136(2): e448-62 Disponible en: https://pubmed.ncbi.nlm.nih. gov/26152671/

6. Zengin-Akkuş P, Çelen-Yoldaş T, Kurtipek G, Nursel Özmert E. Speech delay in toddlers: Are they only "late talkers"? Turk. J. Pediatr [Internet]. 2018 [citado junio 2021]; 60: 165-172. Disponible en: https://www.turkishjournalpediatrics.org/uploads/pdf_TJP_1824.pdf

7. Del Rincón PLN. Prevalencia de los trastornos del lenguaje y su impacto en la familia. En: Psicología de la Familia: estructuras y Trastornos: Estudios en Homenaje al Profesor Francisco Molina. 2014:149-158.

8. Blumenfeld A, Carrizo Olalla J, D’Angelo SI, González NS, Sadras Y, Graizer $\mathrm{S}$, et al. Retraso del desarrollo del lenguaje en niños de 24 meses en un centro de salud en la Ciudad de Buenos Aires. Arch Argent Pediatr [Internet]. 2018 [citado marzo 2021];116(4):242-247. Disponible en: https://www.sap.org.ar/uploads/archivos/general/files_ao_blumenfeld_46pdf_1528145127.pdf

9. Galdámez GM, Castellanos N, Rodríguez IS, Sierra JE, Alger J, Gonzales $\mathrm{RM}$, et al. High prevalence of disability in children aged 2-17 years old In Honduras, Central America, 2017: Abstracts of scientific papers and posters presented at the ISPRM world congress and annual meeting of the association of academic physiatrists Orlando, Florida march 4-9, 2020. J Int Soc Phys Rehabil Med [Internet] 2020 [citado marzo 2020];3, Suppl S1:1488. Disponible en: http://www.jisprm.org/text. asp?2020/3/5/1/283766

10. Besoain-Saldaña A, Rebolledo Sanhueza J, Manríquez Hizaut M, Cortínez Rojas V, Huepe Ortega G, Aliaga Castillo V. Rehabilitación Basada en la Comunidad (RBC) en centros de atención primaria en Chile. Rev Saude Pública. [Internet]. 2020 [citado marzo 2021];54:38. Disponible en: https:// www.scielo.br/j/rsp/a/fct9bVSqFKkx6qW4FMZVYQD/?format=pdf\&lang= es

11. Muñoz Borja P. Guías didácticas para el desarrollo de la estrategia de Rehabilitación Basada en la Comunidad[Internet]. Cali: Ministerio de Educación Nacional; 2016 [citado julio 2021]. Disponible en: https://www.researchgate.net/publication/301614478_Guias_didacticas_para_el_desarrollo_de_la_estrategia_de_Rehabilitacion_Basada_en_la_Comunidad

12. Nelson HD, Nygren P, Walker M, Panoscha R. Screening for speech and language delay in preschool children: systematic evidence review for the US Preventive Services Task Force. Pediatrics [Internet]. 2006 [citado abril 2020];117(2): e298-319. Disponible en: https://pubmed.ncbi.nlm.nih. gov/16452337/

13. Jullien S. Screening for language and speech delay in children un- 
der five years. BMC Pediatrics [Internet]. 2021 [citado agosto 2021]; 21(Supl 1):362. Disponible en: https://www.ncbi.n/m.nih.gov/pmc/articles/ PMC8424786/pdf/12887_2021_Article_2817.pdf

14. Sunderajan T, Kanhere SV. Speech and language delay in children: Prevalence and risk factors. J Family Med Prim Care [Internet]. 2019 [citado en septiembre 2021];8(5):1642-1646. Disponible en: https://www.ncbi.nlm. nih.gov/pmc/articles/PMC6559061/

15. Dale PS, McMillan AJ, Hayiou-Thomas ME, Plomin R. Illusory recovery: are recovered children with early language delay at continuing elevated risk? Am J Speech Lang Pathol [Internet]. 2014 [citado agosto 2021];23(3):437447. Disponible en: https://pubmed.ncbi.nlm.nih.gov/24686486/

16. Dale PS, Patterson JL. Early identification of language delay. En: Encyclopedia of Early Childhood Development [Internet]. 2017 [citado julio 2021]. Disponible en: https://www.child-encyclopedia.com/pdf/expert/languagedevelopment-and-literacy/according-experts/early-identification-languagedelay

17. Zwarenstein M, Treweek S, Gagnier JJ, Altman DG, Tunis S, Haynes B, et al. Improving the reporting of pragmatic trials: an extension of the CONSORT statement. BMJ [Internet]. 2008 [citado marzo 2020];337: a2390. Disponible en: https://www.bmj.com/content/337/bmj.a2390

18. Roberts MY, Kaiser AP. Early intervention for toddlers with language delays: a randomized controlled trial. Pediatrics [Internet]. 2015 [citado marzo 2020];135(4):686-93. Disponible en: https://pubmed.ncbi.nlm.nih. gov/25733749/

19. Azcoaga, JE, Bello JA, Citrinovitz J, Derman B, Frutos WM. Los retardos en el lenguaje del niño. Barcelona: Paidos. 1981. Cap 11.

20. Roberts MY, Kaiser AP. The effectiveness of parent-implemented language interventions: a meta-analysis. Am J Speech Lang Pathol [Internet]. 2011[citado abril 2020];20(3):180-199. Disponible en: https://pubmed. ncbi.nlm.nih.gov/21478280/

21. Law J, Garrett Z, Nye C. The efficacy of treatment for children with developmental speech and language delay/disorder: a meta-analysis. J Speech, Lang Hear Res [Internet]. 2004 [citado noviembre 2020];47(4):924-943. Disponible en: https://pubmed.ncbi.nlm.nih.gov/15324296/

22. Berkman ND, Wallace I, Watson L, Coyne-Beasley T, Cullen K, Wood C, Lohr KN. Screening for Speech and Language Delays and Disorders in Children Age 5 Years or Younger: A Systematic Review for the U.S. Preventive Services Task Force. Evidence Synthesis [Internet] 2015 [citado diciembre 2020]; 20: 47-48. Disponible en:https://www.ncbi.nlm.nih.gov/ books/NBK305674/pdf/Bookshelf_NBK305674.pdf

23. Buschmann A, Jooss B, Rupp A, Feldhusen F, Pietz J, Philippi H. Parent based language intervention for 2-year-old children with specific expressive language delay: a randomised controlled trial. Arch Dis Child [Internet]. 2009 [citado diciembre 2020];94(2):110-116. Disponible en: https://pubmed.ncbi.nlm.nih.gov/18703544/

24. Organización Panamericana de la Salud. Informes de la situación de la COVID-19. Washington DC: OPS/OMS [Internet]. 2020 [citado enero 2021]. Disponible en: https://www.paho.org/es/informes-situacion-covid-19
ABSTRACT. Background: In Honduras, $28 \%$ of children with a disability have a functional alteration related to voice and speech. The Community Based Rehabilitation (CBR) is a strategy to improve the access to rehabilitation Objective: Compare the effectiveness of language therapies with CBR focus versus conventional therapy in children with Language Speech Delay (LSD) in Specialized Center for Physical Medicine and Rehabilitation, Instituto Hondureño de Seguridad Social (IHSS), Honduras in June 2019 - January 2020. Methods: Randomized controlled trial. Children of 2 to 5 years of age with LSD were randomized to intervention group (CBR) and control group. Intervention consisted in training the parents so they could do the therapies to their children at home. Study population was characterized, and a Cox regression model was performed. Results: 48 children completed 2 evaluations.Median age of 3 years, $77.1 \%$ between 2 and 3 years, male predominance with $79.2 \%$, urban provenance with $82.9 \%$, high education level of the mother of $47.9 \%$. Children in CBR had $4.7 \%$ more probability of improving unadjusted Hazard Ratio (HR) (1.047, $p=0.889, \mathrm{Cl} 95 \%$ $0.5434-2.0194)$; when adjusted the probability of improving was $10.2 \%$ (adjusted HR 1.1016, $p=0.783, \mathrm{Cl} 95 \% 0.55-2.1966$ ); none of the models reached statistical significance. Conclusions: No difference was found in the effectiveness between the groups; the use of CBR therapies may decrease costs for the patient's parents and for the IHSS.

Keywords: Community-Based participatory Research; Disabled children; Rehabilitation. 